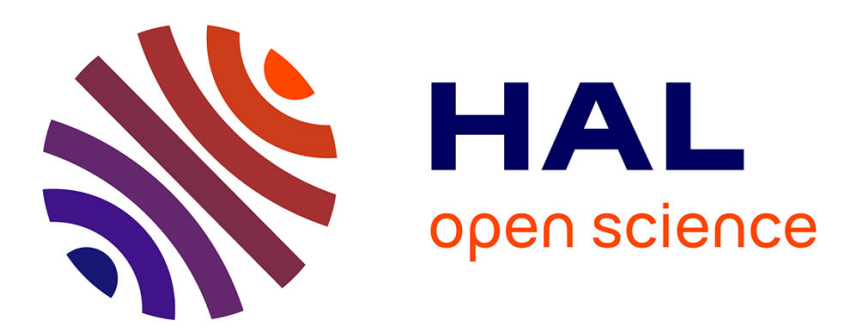

\title{
Homogenizing media containing a highly conductive honeycomb substructure
}

\author{
Isabelle Gruais, Dan Polisevski
}

\section{To cite this version:}

Isabelle Gruais, Dan Polisevski. Homogenizing media containing a highly conductive honeycomb substructure. Asymptotic Analysis, 2010, 67 (1-2), pp.33-43. hal-00380915

\section{HAL Id: hal-00380915 https://hal.science/hal-00380915}

Submitted on 4 May 2009

HAL is a multi-disciplinary open access archive for the deposit and dissemination of scientific research documents, whether they are published or not. The documents may come from teaching and research institutions in France or abroad, or from public or private research centers.
L'archive ouverte pluridisciplinaire HAL, est destinée au dépôt et à la diffusion de documents scientifiques de niveau recherche, publiés ou non, émanant des établissements d'enseignement et de recherche français ou étrangers, des laboratoires publics ou privés. 


\title{
Homogenizing media containing a highly conductive honeycomb substructure
}

\author{
by Isabelle Gruais * and Dan Poliševski **
}

\begin{abstract}
The present paper deals with the homogenization of the heat conduction which takes place in a binary three-dimensional medium consisting of an ambiental phase having conductivity of unity order and a rectangular honeycomb structure formed by a set of thin layers crossing orthogonally and periodically. We consider the case when the conductivity of the thin layers is in inverse proportion to the vanishing volume of the rectangular honeycomb structure. We find the system that governs the asymptotic behaviour of the temperature distribution of this binary medium. The dependence with respect to the thicknesses of the layers is also emphasized. We use an energetic method associated to a natural control-zone of the vanishing domain.
\end{abstract}

Mathematical Subject Classification (2000). 35B27, 35K57, 76R50.

Keywords. homogenization, conduction, fine-scale, honeycomb structure.

\section{Introduction}

The study of the lattice-type structures which are characterized by periodicity and small thickness of the material is one of the main achievements of the homogenization theory. Civil engineering, electrotechnics and the aerospace industry are mainly concerned by composite materials, particularly truss structures, which have to be treated by techniques of homogenization when direct computations fail.

The foundations of homogenized layered materials were laid down by Murat and Tartar in their pioneering work [10]. This method was still used by [9] in the framework of weaker topologies. The case of $B V$-functions and sequences of measures is worked out in [6]. Many examples and applications may be found in [1. The difficulties arising from the direct computation of the behaviour of these structures are twofold: the great number of cells and the small thickness of the material. The periodic distribution classically suggests that the homogenization method for perforated domains should be used as in [11], where the period $\varepsilon$ and the thickness $\delta$ of the material are considered as independent vanishing parameters. A classical issue is then to consider the problem of permuting both converging processes. This is made in details in 11. As the pratical point of view favors geometric consideration, measures provide a more general tool when 
they are confined to the description of the critical part of a system, as in [2] and [3]. This approach was used in [12], where the homogenization of elastic reticulated structures is performed for an anisotropic material surrounded by an empty environment.

Our study is based on the control-zone method which was introduced and developed by [4]-[5] and [7]-8], specific to the binary composition of the system, namely the truss and the ambiental phase. This procedure proved its efficiency in the modelling of fine substructures where concentrated material of high contribution influences the behavior of the global problem in spite of its vanishing volume. The asymptotic treatment reveals the apparent paradox between an obviously disappearing element and its everlasting action on their environment. The coupling between both components manifests itself through the rarefying ratio $\gamma_{\varepsilon}$ which arises as a criterium for the reduced problem to exist. Unlike the critical case studied in [5], the connections between the layers of the reticulated structure annihilate the capacitary term $\lim _{\varepsilon \rightarrow 0} \gamma_{\varepsilon}$ when it is defined. This was also observed in [8] in the absence of connections. Interestingly, the presence of the ambiental phase substantially modifies the contribution of the truss in a way that cannot be anticipated from [1]-12], because the sequence of problems under consideration behaves singularly with respect to the period of distribution and involves a new criterium, namely the capacity of the intersections of the crossing layers.

The present paper is organized as follows:

Section 2 is devoted to the main notations and to the description of the initial problem. We set the functional framework for which the existence and uniqueness of the solution can be established.

In the first part of Section 3 we present two operators that have a localizing effect and that we use in order to obtain the specific inequalities related to honeycomb structures. We also define the capacitary functions and step approximation operators which are associated with the test-functions defined in our control-zones. They overcome the singular behavior of the energy term when $\varepsilon$ tends to zero.

Section 4 deals with the homogenization process in the reticulated case in the so-called box structure geometry. We obtain the homogenized equation which displays explicit effective coefficients. The proof relies on the energetic method applied in the control-zone context. No critical thickness of the layers appears and the intersections of the layers have no distinct influence upon the asymptotic behaviour of the temperature.

The homogenization process in the gridwork case is worked out in Section 5 . We obtain the homogenized equation and explicit effective coefficients in this case also. The proofs are only sketched because the arguments follow the same lines as in Section 4 .

The significant difference between the effective coefficients obtained in the two cases studied here shows how important is the internal geometry of the vanishing superconductive material. 


\section{Setting of the problem}

Let $\left.\Omega=I^{3}, I=\right]-\frac{1}{2}, \frac{1}{2}[$ and $n \in \mathrm{N}$. From now on we use the notations

$$
\varepsilon=\frac{1}{2 n+1}, \quad \mathbf{Z}_{\varepsilon}=\{k \in \mathbf{Z}, \quad|k| \leq n\} \quad \text { and } \quad I_{\varepsilon}^{k}=\varepsilon k+\varepsilon I, \quad k \in \mathbf{Z}_{\varepsilon} .
$$

Obviously, $\operatorname{card} \mathbf{Z}_{\varepsilon}=1 / \varepsilon$ and $x \in \bar{I}$ if and only if there exists $k \in \mathbf{Z}_{\varepsilon}$ such that $x \in \bar{I}_{\varepsilon}^{k}$.

For any $i \in\{1,2,3\}$, we consider $r_{\varepsilon}^{i} \geq 0, r_{\varepsilon}^{i}<<\varepsilon$, that is $\frac{r_{\varepsilon}^{i}}{\varepsilon} \rightarrow 0$ when $\varepsilon \rightarrow 0$. For any $k \in \mathbf{Z}_{\varepsilon}$ we define:

$$
\begin{gathered}
T_{\varepsilon, k}^{i}=\left\{x=\left(x_{1}, x_{2}, x_{3}\right) \in \Omega,\left|x_{i}-\varepsilon k\right|<r_{\varepsilon}^{i}\right\}, T_{\varepsilon}^{i}=\cup_{k \in \mathbf{Z}_{\varepsilon}} T_{\varepsilon, k}^{i} \\
T_{\varepsilon}=\cup_{i=1}^{3} T_{\varepsilon}^{i}, \quad T_{\varepsilon}^{i j}=T_{\varepsilon}^{i} \cap T_{\varepsilon}^{j}, \quad \text { for } \quad 1 \leq i<j \leq 3 .
\end{gathered}
$$

We consider that $\Omega$ is occupied by two materials with highly different conductivities; one is highly conductive and it is concentrated in the vanishing domain of the thin layers $T_{\varepsilon}\left(\left|T_{\varepsilon}\right| \rightarrow 0\right)$ and the other forms the ambiental phase which has conductivity of unity order.

Assuming that we are given a source term $f_{\varepsilon}$ in $\Omega$ and some conductivity coefficients $a, b>0$, we consider the heat conduction problem, that is we study the temperature $u_{\varepsilon}$ which satisfies in some sense the system

$$
\begin{gathered}
-a \Delta u_{\varepsilon}=f_{\varepsilon} \quad \text { in } \quad \Omega \backslash T_{\varepsilon} \\
-\frac{b}{\left|T_{\varepsilon}\right|} \Delta u_{\varepsilon}=f_{\varepsilon} \quad \text { in } T_{\varepsilon} \\
u_{\varepsilon}=0 \text { on } \partial \Omega,
\end{gathered}
$$

together with the natural transmission conditions on the interface $\partial T_{\varepsilon} \backslash \partial \Omega$.

More precisely, assuming that $f_{\varepsilon} \in H^{-1}(\Omega)$, the variational formulation of our problem is :

To find $u_{\varepsilon} \in H_{0}^{1}(\Omega)$ such that

$$
a \int_{\Omega \backslash T_{\varepsilon}} \nabla u_{\varepsilon} \nabla v+b f_{T_{\varepsilon}} \nabla u_{\varepsilon} \nabla v=\left\langle f_{\varepsilon}, v\right\rangle, \quad \forall v \in H_{0}^{1}(\Omega),
$$

where we have used the notation

$$
f_{E} \cdot=\frac{1}{|E|} \int_{E} \cdot \quad \text { for any measurable } E \subset \Omega
$$

and $\langle\cdot, \cdot\rangle$ is the duality product between $H^{-1}(\Omega)$ and $H_{0}^{1}(\Omega)$.

Applying the Lax-Milgram theorem we obtain:

Proposition 2.1 The variational equation (円) has a unique solution $u_{\varepsilon} \in H_{0}^{1}(\Omega)$. 
The aim of this paper is to describe the asymptotic behaviour of the temperature $u_{\varepsilon}$ as $\varepsilon \rightarrow 0$, assuming that the source term is weakly convergent:

$$
\exists f \in H^{-1}(\Omega) \text { such that } f_{\varepsilon} \rightarrow f \text { in } H^{-1}(\Omega) .
$$

We have to remark here that the boundedness of $\left(f_{\varepsilon}\right)_{\varepsilon}$ implies immediately:

Proposition $2.2\left(u_{\varepsilon}\right)_{\varepsilon}$ is bounded in $H_{0}^{1}(\Omega)$ and there exists $C>0$, independent of $\varepsilon$, such that

$$
f_{T_{\varepsilon}}\left|\nabla u_{\varepsilon}\right|^{2} \leq C
$$

\section{The control-zone homogenization method}

In order to obtain further results, specific to the thin substructure considered here, we have to introduce the following operators:

Definition 3.1 To any $u \in H^{1}(\Omega)$ we associate $G_{\varepsilon}^{i}(u) \in L^{2}(\Omega)$ defined by the following:

$$
\begin{gathered}
G_{\varepsilon}^{i}(u)\left(x_{1}, x_{2}, x_{3}\right)=\sum_{k \in \mathbf{Z}_{\varepsilon}} G_{\varepsilon, k}^{i}(u)\left(\bar{x}_{i}\right) 1_{I_{\varepsilon}^{k}}\left(x_{i}\right), \quad \bar{x}_{i}=\left(\cdots, \not x_{i}, \cdots\right) \in I^{2} \\
G_{\varepsilon, k}^{i}(u)=\left.\frac{1}{2} u\right|_{x_{i}=\varepsilon k-r_{\varepsilon}^{i}}+\left.\frac{1}{2} u\right|_{x_{i}=\varepsilon k+r_{\varepsilon}^{i}}, \quad k \in \mathbf{Z}_{\varepsilon}
\end{gathered}
$$

where $\left.u\right|_{x_{i}=\varepsilon k \pm r_{\varepsilon}^{i}}$ are the traces of $u$ on the corresponding faces of $T_{\varepsilon, k}^{i}$. [8:

These operators have three basic properties, which were already proved in

Proposition 3.2 For any $u \in H^{1}(\Omega)$ and $i \in\{1,2,3\}$, we have

$$
\begin{gathered}
f_{T_{\varepsilon}^{i}}\left|G_{\varepsilon}^{i}(u)-u\right|^{2} \leq r_{\varepsilon}^{i}\left|\frac{\partial u}{\partial x_{i}}\right|_{\Omega}^{2} \\
f_{T_{\varepsilon}^{i}}\left|G_{\varepsilon}^{i}(u)\right|^{2}=\left|G_{\varepsilon}^{i}(u)\right|_{\Omega}^{2} \\
\left|G_{\varepsilon}^{i}(u)-u\right|_{\Omega} \leq \varepsilon\left|\frac{\partial u}{\partial x_{i}}\right|_{\Omega}
\end{gathered}
$$

where $|\cdot|_{\Omega}$ is denoting the norm of $L^{2}(\Omega)$.

The first important consequence is

Theorem 3.3 There exists $C>0$, independent of $\varepsilon$, such that

$$
f_{T_{\varepsilon}}|u|^{2} \leq C|\nabla u|_{\Omega}^{2}, \quad \forall u \in H_{0}^{1}(\Omega) .
$$


Proof. Let us notice that

$$
f_{T_{\varepsilon}}|u|^{2} \leq \sum_{i=1}^{2} f_{T_{\varepsilon}^{i}}|u|^{2} \leq 2 \sum_{i=1}^{2} f_{T_{\varepsilon}^{i}}\left(\left|G_{\varepsilon}^{i}(u)-u\right|^{2}+\left|G_{\varepsilon}^{i}(u)\right|^{2}\right) .
$$

Using (13)-(14), it yields

$$
f_{T_{\varepsilon}}|u|^{2} \leq 2\left(\max _{i=1,2,3} r_{\varepsilon}^{i}\right)|\nabla u|_{\Omega}^{2}+4 \sum_{i=1}^{2}\left(\left|G_{\varepsilon}^{i}(u)-u\right|_{\Omega}^{2}+|u|_{\Omega}^{2}\right)
$$

and the proof is completed by 10 ) and the Poincaré-Friedrichs inequality in $\Omega$.

As the techniques of Section 3 [5] can be used to the domain $T_{\varepsilon}^{i j}(i<j)$, then, according to [2], we have:

Theorem 3.4 There exists $C>0$, independent of $\varepsilon$, such that

$$
f_{T_{\varepsilon}^{i j}}|u|^{2} \leq C \max \left(1, \varepsilon^{2} \ln \frac{1}{r_{\varepsilon}^{i}}, \varepsilon^{2} \ln \frac{1}{r_{\varepsilon}^{j}}\right)|\nabla u|_{\Omega}^{2}, \quad \forall u \in H_{0}^{1}(\Omega) .
$$

Finally, we remind an estimation of the same type proved in [5] and which is associated to

$$
T_{\varepsilon}^{0}=\cap_{i=1}^{3} T_{\varepsilon}^{i} .
$$

Theorem 3.5 There exists $C>0$, independent of $\varepsilon$, such that

$$
f_{T_{\varepsilon}^{0}}|u|^{2} \leq C \max \left(1, \frac{\varepsilon^{3}}{r_{\varepsilon}}\right)|\nabla u|_{\Omega}^{2}, \quad \forall u \in H_{0}^{1}(\Omega) .
$$

As the vanishing highly conductive layers have thicknesses of size $r_{\varepsilon}^{i}, 0<$ $r_{\varepsilon}^{i}<<\varepsilon$, we find the asymptotic behaviour of $u_{\varepsilon}$ by applying a control-zone method, that is an energetic method using test-functions associated to an adequate control-zone: a vanishing set which includes the layers and has much larger thicknesses $R_{\varepsilon}^{i}$, where $r_{\varepsilon}^{i}<<R_{\varepsilon}^{i}<<\varepsilon$.

First, let us introduce the tools of this method. Denoting

$$
\mathcal{R}=\left\{\left(R_{\varepsilon}\right)_{\varepsilon}, r_{\varepsilon}<<R_{\varepsilon}<<\varepsilon\right\},
$$

then, for any $\left(R_{\varepsilon}^{i}\right)_{\varepsilon} \in \mathcal{R}$ we define the control-zone of the present problem by

$$
\mathcal{C}_{\varepsilon}=\cup_{i=1}^{3} \mathcal{C}_{\varepsilon}^{i}, \quad \mathcal{C}_{\varepsilon}^{i}=\cup_{k \in \mathbf{Z}_{\varepsilon}} \mathcal{C}_{\varepsilon, k}^{i}, \quad i \in\{1,2,3\},
$$

where for any $k \in \mathbf{Z}_{\varepsilon}$ and $i \in\{1,2,3\}$ we have

$$
\mathcal{C}_{\varepsilon, k}^{i}=\left\{x=\left(x_{1}, x_{2}, x_{3}\right) \in \Omega, \quad\left|x_{i}-\varepsilon k\right|<R_{\varepsilon}^{i}\right\} .
$$

The test-functions associated to this control-zone are defined by using the following capacitary functions $w_{\varepsilon}^{i} \in W^{1, \infty}(\Omega)(i=1,2,3)$, given by

$$
w_{\varepsilon}^{i}\left(x_{1}, x_{2}, x_{3}\right)= \begin{cases}1-\frac{r_{\varepsilon}^{i}}{R_{\varepsilon}^{i}} & \text { if } \quad x=\left(x_{1}, x_{2}, x_{3}\right) \in T_{\varepsilon}^{i} \\ 1-\frac{\left|x_{i}-\varepsilon k\right|}{R_{\varepsilon}^{i}} & \text { if } \quad x \in\left(\mathcal{C}_{\varepsilon, k}^{i} \backslash T_{\varepsilon, k}^{i}\right) \text { for some } k \in \mathbf{Z}_{\varepsilon} \\ 0 & \text { if } \quad x \in \Omega \backslash \mathcal{C}_{\varepsilon}^{i},\end{cases}
$$


and the step approximation operators introduced by

Definition 3.6 To any $\varphi \in \mathcal{D}(\Omega)$ we associate $\varphi_{\varepsilon}^{i} \in L^{\infty}(\Omega) \quad(i=1,2,3)$, as follows

$$
\varphi_{\varepsilon}^{i}\left(x_{1}, x_{2}, x_{3}\right)=\left.\sum_{k \in \mathbf{Z}_{\varepsilon}} \varphi\right|_{x_{i}=\varepsilon k}\left(\bar{x}_{i}\right) 1_{I_{R_{\varepsilon}^{i}}^{k}}\left(x_{i}\right),
$$

where $I_{R_{\varepsilon}^{i}}^{k}:=\varepsilon k+2 R_{\varepsilon}^{i} I$.

These operators have the following basic properties:

$$
\begin{gathered}
\left|\nabla w_{\varepsilon}^{i}\right|_{\mathcal{C}_{\varepsilon}} \leq\left(\frac{2}{\varepsilon R_{\varepsilon}^{i}}\right)^{1 / 2} \\
\left|\varphi-\varphi_{\varepsilon}^{i}\right|_{L^{\infty}\left(\mathcal{C}_{\varepsilon}^{i}\right)} \leq R_{\varepsilon}^{i}|\nabla \varphi|_{L^{\infty}(\Omega)} . \\
\left|\nabla \varphi_{\varepsilon}^{i}\right|_{\mathcal{C}_{\varepsilon}} \leq\left(\frac{2 R_{\varepsilon}^{i}}{\varepsilon}\right)^{1 / 2}|\nabla \varphi|_{L^{\infty}(\Omega)} .
\end{gathered}
$$

\section{The reticulated case}

For any $i \in\{1,2,3\}$ let us denote $m_{i} \geq 0$ as the limit of

$$
m_{i}=\lim _{\varepsilon \rightarrow 0} \frac{\left|T_{\varepsilon}^{i}\right|}{\left|T_{\varepsilon}\right|}
$$

Obviously, we have

$$
m_{1}+m_{2}+m_{3}=1 .
$$

In this section, we consider the case when

$$
m_{i}>0, \quad \forall i \in\{1,2,3\},
$$

that is the case when all the three parameters $r_{\varepsilon}^{i}$ have the same order of magnitude with respect to $\varepsilon$. This geometry is called sometimes as the box-structure case.

We can present now the preliminary convergence results:

Proposition 4.1 There exists $u \in H_{0}^{1}(\Omega)$ such that, on some subsequence, there hold

$$
\begin{gathered}
u_{\varepsilon} \rightarrow u \quad \text { in } \quad H_{0}^{1}(\Omega) \\
G_{\varepsilon}^{i}\left(u_{\varepsilon}\right) \rightarrow u \quad \text { in } L^{2}(\Omega), \quad \forall i \in\{1,2,3\} \\
f_{T_{\varepsilon}} u_{\varepsilon} v \rightarrow \int_{\Omega} u v, \quad \forall v \in H_{0}^{1}(\Omega) .
\end{gathered}
$$

Moreover, for any $i \in\{1,2,3\}$ we have:

$$
f_{T_{\varepsilon}^{i}} \frac{\partial u_{\varepsilon}}{\partial x_{j}} v \rightarrow \int_{\Omega} \frac{\partial u}{\partial x_{j}} v, \quad \forall v \in H_{0}^{1}(\Omega), \quad \forall j \in\{1,2,3\}, \quad j \neq i .
$$


Proof. The weak convergence (30) follows from Proposition 2.2. As $H_{0}^{1}(\Omega)$ is compactly embedded in $L^{2}(\Omega)$, the strong convergence (31) is obtained by using (15) and (30).

In order to prove (32) we remark that

$$
f_{T_{\varepsilon}} u_{\varepsilon} v=\frac{\left|T_{\varepsilon}^{0}\right|}{\left|T_{\varepsilon}\right|} f_{T_{\varepsilon}^{0}} u_{\varepsilon} v-\sum_{1 \leq i<j \leq 3} \frac{\left|T_{\varepsilon}^{i j}\right|}{\left|T_{\varepsilon}\right|} f_{T_{\varepsilon}^{i j}} u_{\varepsilon} v+\sum_{i=1}^{3} \frac{\left|T_{\varepsilon}^{i}\right|}{\left|T_{\varepsilon}\right|} f_{T_{\varepsilon}^{i}} u_{\varepsilon} v .
$$

Using (10), (17) and (19), we prove that the first two terms of (34) are converging to zero, as follows:

$$
\begin{gathered}
\left|\frac{\left|T_{\varepsilon}^{0}\right|}{\left|T_{\varepsilon}\right|} f_{T_{\varepsilon}^{0}} u_{\varepsilon} v\right| \leq C \frac{\left|T_{\varepsilon}^{0}\right|}{\left|T_{\varepsilon}\right|} \max \left(1, \frac{\varepsilon^{3 / 2}}{r_{\varepsilon}^{1 / 2}}\right)|\nabla v|_{\Omega} \rightarrow 0, \\
\left|\frac{\left|T_{\varepsilon}^{i j}\right|}{\left|T_{\varepsilon}\right|} f_{T_{\varepsilon}^{i j}} u_{\varepsilon} v\right| \leq C \frac{\left|T_{\varepsilon}^{i j}\right|}{\left|T_{\varepsilon}\right|} \max \left(1, \varepsilon \ln ^{\frac{1}{2}} \frac{1}{r_{\varepsilon}^{i}}, \varepsilon \ln ^{\frac{1}{2}} \frac{1}{r_{\varepsilon}^{j}}\right)|\nabla v|_{\Omega} \rightarrow 0 .
\end{gathered}
$$

Taking into account (28), the proof of (32) is completed by applying to Proposition 2.15 of 8 , where we have proved that

$$
f_{T_{\varepsilon}^{i}} u_{\varepsilon} v \rightarrow \int_{\Omega} u v, \quad \forall v \in H_{0}^{1}(\Omega) .
$$

Next, let $\varphi \in \mathcal{D}(\Omega)$ and $i \in\{1,2,3\}$. Denoting by $\nu=\left(\nu_{1}, \nu_{2}, \nu_{3}\right)$ the outward normal to $\partial T_{\varepsilon}$ we obviously have $\varphi \nu_{j}=0$ on $\partial T_{\varepsilon}^{i}(j \neq i)$ and hence:

$$
f_{T_{\varepsilon}^{i}} \frac{\partial u_{\varepsilon}}{\partial x_{j}} \varphi=-f_{T_{\varepsilon}^{i}} u_{\varepsilon} \frac{\partial \varphi}{\partial x_{j}} .
$$

Using (35), from the previous relation it follows:

$$
f_{T_{\varepsilon}^{i}} \frac{\partial u_{\varepsilon}}{\partial x_{j}} \varphi \rightarrow-\int_{\Omega} u \frac{\partial \varphi}{\partial x_{j}}=\int_{\Omega} \frac{\partial u}{\partial x_{j}} \varphi .
$$

The proof of 33 is completed by continuity, using (10) and (16).

Now we can present our main result.

Theorem $4.2\left(u_{\varepsilon}\right)_{\varepsilon}$ is weakly convergent in $H_{0}^{1}(\Omega)$. Its limit, $u \in H_{0}^{1}(\Omega)$, is the only solution of the equation

$$
-\sum_{i=1}^{3}\left(a+\frac{b}{3}\left(1-m_{i}\right)\right) \frac{\partial^{2} u}{\partial x_{i}^{2}}=f \quad \text { in } \Omega .
$$

Proof. For any $i \in\{1,2,3\}$, let $\left(\mathbf{R}_{\varepsilon}^{i}\right)_{\varepsilon} \in \mathcal{R}$ and $\varphi \in \mathcal{D}(\Omega)$. Using the definitions (23)-24), we denote

$$
v_{\varepsilon}(\varphi)=\sum_{i=1}^{3}\left(\left(1-\frac{r_{\varepsilon}^{i}}{R_{\varepsilon}^{i}}\right) \varphi+\left(\varphi_{\varepsilon}^{i}-\varphi\right) w_{\varepsilon}^{i}\right) .
$$


We notice that

$$
\varphi_{\varepsilon}^{i}(x) w_{\varepsilon}^{i}(x)=\left\{\begin{array}{l}
\left.\left(1-\frac{r_{\varepsilon}^{i}}{R_{\varepsilon}^{i}}\right) \varphi\right|_{x_{i}=\varepsilon k}\left(\bar{x}_{i}\right), x \in T_{\varepsilon, k}^{i} \quad \text { for some } k \in \mathbf{Z}_{\varepsilon} \\
\left.\left(1-\frac{\left|x_{i}-\varepsilon k\right|}{R_{\varepsilon}^{i}}\right) \varphi\right|_{x_{i}=\varepsilon k}\left(\bar{x}_{i}\right), x \in\left(\mathcal{C}_{\varepsilon, k}^{i} \backslash T_{\varepsilon, k}^{i}\right), k \in \mathbf{Z}_{\varepsilon} \\
0, x \in \Omega \backslash \mathcal{C}_{\varepsilon}^{i}
\end{array}\right.
$$

and hence $v_{\varepsilon}(\varphi) \in W_{0}^{1, \infty}(\Omega)$. Then we set $v=v_{\varepsilon}(\varphi)$ in (7) and it follows

$$
\begin{aligned}
a \sum_{i=1}^{3}\left(1-\frac{r_{\varepsilon}^{i}}{R_{\varepsilon}^{i}}\right) \int_{\Omega \backslash \mathcal{C}_{\varepsilon}} \nabla u_{\varepsilon} \nabla \varphi+a \int_{\mathcal{C}_{\varepsilon} \backslash T_{\varepsilon}} \nabla u_{\varepsilon} \nabla v_{\varepsilon}(\varphi)+ \\
+b \sum_{i=1}^{3}\left(1-\frac{r_{\varepsilon}^{i}}{R_{\varepsilon}^{i}}\right) f_{T_{\varepsilon}} \nabla u_{\varepsilon} \nabla \varphi_{\varepsilon}^{i}=\left\langle f_{\varepsilon}, v_{\varepsilon}(\varphi)\right\rangle .
\end{aligned}
$$

As $\left|\mathcal{C}_{\varepsilon}\right| \rightarrow 0$ it follows that

$$
\left(1-\frac{r_{\varepsilon}^{i}}{R_{\varepsilon}^{i}}\right)(\nabla \varphi) 1_{\Omega \backslash \mathcal{C}_{\varepsilon}} \rightarrow \nabla \varphi \quad \text { strongly in } \quad L^{2}(\Omega)
$$

and using (30) we obtain the convergence of the first left-hand side term of (39):

$$
a \sum_{i=1}^{3}\left(1-\frac{r_{\varepsilon}^{i}}{R_{\varepsilon}^{i}}\right) \int_{\Omega \backslash \mathcal{C}_{\varepsilon}} \nabla u_{\varepsilon} \nabla \varphi \rightarrow 3 a \int_{\Omega} \nabla u \nabla \varphi .
$$

The second left-hand side term of (39) is converging to zero. Indeed, as $\left(u_{\varepsilon}\right)_{\varepsilon}$ is bounded in $H_{0}^{1}(\Omega)$, we have

$$
\left|a \int_{\mathcal{C}_{\varepsilon} \backslash T_{\varepsilon}} \nabla u_{\varepsilon} \nabla v_{\varepsilon}(\varphi)\right| \leq C\left|\nabla v_{\varepsilon}(\varphi)\right|_{\mathcal{C}_{\varepsilon}} .
$$

Moreover, by a straightforward computation we find that

$$
\left|\nabla v_{\varepsilon}(\varphi)\right|_{\mathcal{C}_{\varepsilon}} \leq C|\nabla \varphi|_{\mathcal{C}_{\varepsilon}}+C \sum_{i=1}^{3}\left(\left.\left|\varphi_{\varepsilon}^{i}-\varphi\right|_{L^{\infty}\left(\mathcal{C}_{\varepsilon}^{i}\right)}\left|\nabla w_{\varepsilon}^{i}\right|\right|_{\mathcal{C}_{\varepsilon}}+\left|\nabla_{\bar{x}_{i}} \varphi_{\varepsilon}^{i}\right|_{\mathcal{C}_{\varepsilon}}\right)
$$

and the assertion is proved by using (25) $-(27)$ :

$$
\left|\nabla v_{\varepsilon}(\varphi)\right|_{\mathcal{C}_{\varepsilon}} \leq C|\nabla \varphi|_{L^{\infty}(\Omega)} \sum_{i=1}^{3}\left(\frac{R_{\varepsilon}^{i}}{\varepsilon}\right)^{1 / 2} \rightarrow 0 .
$$

For the convergence of the third left-hand side term of (39) we notice that

$$
\begin{aligned}
& f_{T_{\varepsilon}} \nabla u_{\varepsilon} \nabla \varphi_{\varepsilon}^{i}=\frac{1}{\left|T_{\varepsilon}\right|} \int_{T_{\varepsilon}^{i}} \nabla u_{\varepsilon} \nabla \varphi_{\varepsilon}^{i}= \\
& \quad=\left.\frac{1}{\left|T_{\varepsilon}\right|} \sum_{k \in \mathbf{Z}_{\varepsilon}} \int_{T_{\varepsilon, k}^{i}} \nabla_{\bar{x}_{i}} u_{\varepsilon}(x) \nabla_{\bar{x}_{i}} \varphi\right|_{x_{i}=\varepsilon k}\left(\bar{x}_{i}\right) d x
\end{aligned} .
$$


Using (10) and the smoothness of $\varphi$ we have

$$
\begin{gathered}
\left|\frac{1}{\left|T_{\varepsilon}\right|} \sum_{k \in \mathbf{Z}_{\varepsilon}} \int_{T_{\varepsilon, k}^{i}} \nabla_{\bar{x}_{i}} u_{\varepsilon}(x)\left(\left.\nabla_{\bar{x}_{i}} \varphi\right|_{x_{i}=\varepsilon k}\left(\bar{x}_{i}\right)-\nabla_{\bar{x}_{i}} \varphi(x)\right) d x\right| \leq \\
\leq C(\varphi) r_{\varepsilon} \frac{1}{\left|T_{\varepsilon}\right|} \int_{T_{\varepsilon}^{i}}\left|\nabla_{\bar{x}_{i}} u_{\varepsilon}\right| \leq C(\varphi) r_{\varepsilon} \rightarrow 0
\end{gathered}
$$

and thus we replace (45) by

$$
\lim _{\varepsilon \rightarrow 0} \int_{T_{\varepsilon}} \nabla u_{\varepsilon} \nabla \varphi_{\varepsilon}^{i}=\lim _{\varepsilon \rightarrow 0} \frac{\left|T_{\varepsilon}^{i}\right|}{\left|T_{\varepsilon}\right|} \int_{T_{\varepsilon}^{i}} \nabla \bar{x}_{i} u_{\varepsilon} \nabla_{\bar{x}_{i}} \varphi .
$$

Next, recalling (28) and applying (33) we obtain

$$
\begin{aligned}
b \sum_{i=1}^{3}\left(1-\frac{r_{\varepsilon}^{i}}{R_{\varepsilon}^{i}}\right) f_{T_{\varepsilon}} \nabla u_{\varepsilon} \nabla \varphi_{\varepsilon}^{i} & \rightarrow b \sum_{i=1}^{3} m_{i} \int_{\Omega} \nabla_{\bar{x}_{i}} u \nabla_{\bar{x}_{i}} \varphi= \\
& =b \sum_{i=1}^{3}\left(1-m_{i}\right) \int_{\Omega} \frac{\partial u}{\partial x_{i}} \frac{\partial \varphi}{\partial x_{i}} .
\end{aligned}
$$

Finally, noticing that (25) and (26) imply

$$
\begin{aligned}
\left|\left\langle f_{\varepsilon},\left(\varphi_{\varepsilon}^{i}-\varphi\right) w_{\varepsilon}^{i}\right\rangle\right| & \leq C\left|w_{\varepsilon}^{i}\right| \mathcal{C}_{\varepsilon}|\nabla \varphi|_{L^{\infty}(\Omega)}+C\left|\nabla w_{\varepsilon}^{i}\right| \mathcal{C}_{\varepsilon}\left|\varphi_{\varepsilon}^{i}-\varphi\right|_{L^{\infty}\left(\mathcal{C}_{\varepsilon}^{i}\right)} \leq \\
& \leq C(\varphi)\left(\left|\mathcal{C}_{\varepsilon}\right|^{1 / 2}+\left|\frac{R_{\varepsilon}^{i}}{\varepsilon}\right|^{1 / 2}\right) \rightarrow 0,
\end{aligned}
$$

we obtain the convergence of the right-hand side term of (39), that is:

$$
\left\langle f_{\varepsilon}, v_{\varepsilon}(\varphi)\right\rangle=\sum_{i=1}^{3}\left(1-\frac{r_{\varepsilon}^{i}}{R_{\varepsilon}^{i}}\right)\left\langle f_{\varepsilon}, \varphi\right\rangle+\sum_{i=1}^{3}\left\langle f_{\varepsilon},\left(\varphi_{\varepsilon}^{i}-\varphi\right) w_{\varepsilon}^{i}\right\rangle \rightarrow 3\langle f, \varphi\rangle .
$$

Resuming, we can say that if we pass (39) to the limit, then we obtain:

$$
3 a \int_{\Omega} \nabla u \nabla \varphi+b \sum_{i=1}^{3}\left(1-m_{i}\right) \int_{\Omega} \frac{\partial u}{\partial x_{i}} \frac{\partial \varphi}{\partial x_{i}}=3\langle f, \varphi\rangle, \quad \forall \varphi \in \mathcal{D}(\Omega),
$$

which is, by continuity, the variational formulation of (36). The convergence of the entire sequence $\left(u_{\varepsilon}\right)_{\varepsilon}$ follows from the unicity of the solution of (36).

Remark 4.3 In spite of the vanishing volume of the rectangular honeycomb structure the homogenized behaviour is anisotropic, except the case when $m_{1}=$ $m_{2}=m_{3}=\frac{1}{3}$.

Remark 4.4 The reticulated domain occupied by all the intersections of the layers has no distinct influence upon the asymptotic distribution of the temper- 
ature. For $i<j$ and $k \in\{i, j\}$, this follows from

$$
\begin{gathered}
\left|\frac{1}{\left|T_{\varepsilon}\right|} \int_{T_{\varepsilon}^{i j}} \nabla u_{\varepsilon} \nabla \varphi_{\varepsilon}^{k}\right| \leq C|\nabla \varphi|_{L^{\infty}(\Omega)} \\
\left(\frac{1}{\left|T_{\varepsilon}\right|} \int_{T_{\varepsilon}^{i j}}\left|\nabla u_{\varepsilon}\right|^{2}\right)^{\frac{1}{2}}\left(\frac{\left|T_{\varepsilon}^{i j}\right|}{\left|T_{\varepsilon}\right|}\right)^{\frac{1}{2}} \leq \\
\leq C(\varphi)\left(\frac{r_{\varepsilon}}{\varepsilon}\right)^{\frac{1}{2}} \rightarrow 0,
\end{gathered}
$$

where we have used (10).

\section{The gridwork case}

Using the notations and definitions of the previous sections, the gridwork case corresponds to the situation when the horizontal layers are missing and the other two layers have the same order of magnitude with respect to $\varepsilon$. It follows that

and there exist

$$
T_{\varepsilon}=T_{\varepsilon}^{1} \cup T_{\varepsilon}^{2}
$$

$$
m_{i}=\lim _{\varepsilon \rightarrow 0} \frac{\left|T_{\varepsilon}^{i}\right|}{\left|T_{\varepsilon}\right|} \quad(i=1,2), \quad \text { such that } \quad m_{1}+m_{2}=1 .
$$

For consistency with the honeycomb case, we also define here

$$
m_{3}=0 .
$$

Obviously, for $i \in\{1,2\}$ th properties of the corresponding operators defined in Section 3 are still valid. Then we prove results similar to those of Proposition 4.1, with only one mentionable distinction:

For any $i \in\{1,2\}$ we have (33).

Consequently, the homogenization result in this case is the following:

Theorem $5.1\left(u_{\varepsilon}\right)_{\varepsilon}$ is weakly convergent in $H_{0}^{1}(\Omega)$. Its limit, $u \in H_{0}^{1}(\Omega)$, is the only solution of the equation:

$$
-\sum_{i=1}^{3}\left(a+\frac{b}{2}\left(1-m_{i}\right)\right) \frac{\partial^{2} u}{\partial x_{i}^{2}}=f \quad \text { in } \quad \Omega .
$$

Proof. The proof is completely analogous to that of Theorem 4.2, with the difference that the control-zones are oriented in only two directions. That is, with the test-function

$$
v_{\varepsilon}(\varphi)=\sum_{i=1}^{2}\left(\left(1-\frac{r_{\varepsilon}^{i}}{R_{\varepsilon}^{i}}\right) \varphi+\left(\varphi_{\varepsilon}^{i}-\varphi\right) w_{\varepsilon}^{i}\right)
$$

we get the same convergence as in (46):

$$
\begin{aligned}
b \sum_{i=1}^{2}\left(1-\frac{r_{\varepsilon}^{i}}{R_{\varepsilon}^{i}}\right) f_{T_{\varepsilon}} \nabla u_{\varepsilon} \nabla \varphi_{\varepsilon}^{i} & \rightarrow b \sum_{i=1}^{2} m_{i} \int_{\Omega} \nabla_{\bar{x}_{i}} u \nabla_{\bar{x}_{i}} \varphi= \\
& =b \sum_{i=1}^{3}\left(1-m_{i}\right) \int_{\Omega} \frac{\partial u}{\partial x_{i}} \frac{\partial \varphi}{\partial x_{i}},
\end{aligned}
$$


where we have taken in account the convention (52) also. From here the proof can be completed identically.

Remark 5.2 Assuming that the quantity $\left|T_{\varepsilon}\right|$ is the same in the both cases that we considered, the significant difference between the equations (36) and (54) shows how important is the internal geometry of the vanishing superconductive material.

Acknowledgements. This work has been accomplished during the visit of D. Polişevschi at the I.R.M.A.R.'s Department of Mechanics (University of Rennes 1), whose support is gratefully acknowledged.

\section{References}

[1] N.S. Bakhvalov, G.P. Panasenko, Homogenization: averaging processes in periodic media, Math. Appl. (Sov. Series), 36, Kluwer Acad. Pub. Group, Dordrecht, 1989.

[2] M. Bellieud, G. Bouchitté, Homogenization of elliptic problems in a fiber reinforced structure. Non local effects, Ann. Scuola Norm. Sup. Pis Cl. Sci.(4), 26(3), 1998, 407-436.

[3] F. Bentalha, I. Gruais, D. Polisevski, Homogenization of a conductive suspension in a Stokes-Boussinesq flow, Applicable Analysis, 85(6-7), 2006, 811-830.

[4] F. Bentalha, I. Gruais, D. Polisevski, Diffusion process in a rarefied binary structure, Revue Roumaine de Mathématiques Pures et Appliquées, 52(1), 2007, 9-34.

[5] F. Bentalha, I. Gruais, D. Polisevski, Diffusion in a highly rarefied binary structure of general periodic shape, Applicable Analysis, 87(6), 2008, 635-655.

[6] G. Bouchitté, C. Picard, Singular perturbations and homogenization in stratified media, Applicable Analysis, 61(3-4), 1996, 307-341.

[7] I. Gruais, D. Polisevsski, Homogenizing a critical binary structure of finite diffusivities, Asymptotic Analysis, 55(1), 2007, 85-102.

[8] I. Gruais, D. Polisevski, Periodic structures separated by highly conductive thin layers, Prépublication IRMAR, 08-28, 2008, 1-21.

[9] B. HÉron, J. Mossino, C. Picard, Homogenization of some quasilinear problems for stratified media with low and high conductivities, Differential Integral Equations, 7(1), 1994, 157-178. 
[10] F. Murat, L. Tartar, Calcul des variations et homogénéisation, in " Homogenization methods: theory and applications in physics", Collect. Dir. Études Rech. Élec. France, 57, Eyrolles, Paris, 1985, 319-370.

[11] D. Cioranescu, J. Saint-Jean-Paulin, Homogenization of reticulated structures, Springer-Verlag, New-York, 1999.

[12] V.V. Zhikov, S.E. Pastukhova, Derivation of the limit equations of elasticity theory of thin sets, Journal of Mathematical Sciences, 135(1), 2006, 2637-2665.

* Université de Rennes1, I.R.M.A.R, Campus de Beaulieu, 35042 Rennes Cedex (France)

** I.M.A.R., P.O. Box 1-764, RO-014700 Bucharest (Romania). 